\title{
Existence of Nontrivial Solutions and High Energy Solutions for a Class of Quasilinear Schrödinger Equations via the Dual-Perturbation Method
}

\author{
Yu Chen ${ }^{1}$ and Xian $W u^{2}$ \\ ${ }^{1}$ Department of Mathematics, Honghe University, Mengzi, Yunnan 661100, China \\ ${ }^{2}$ Department of Mathematics, Yunnan Normal University, Kunming, Yunnan 650092, China \\ Correspondence should be addressed to Xian Wu; wuxian2001@yahoo.com.cn
}

Received 23 June 2013; Accepted 12 September 2013

Academic Editor: Mihai Mihǎilescu

Copyright (C) 2013 Y. Chen and X. Wu. This is an open access article distributed under the Creative Commons Attribution License, which permits unrestricted use, distribution, and reproduction in any medium, provided the original work is properly cited.

We study the quasilinear Schrödinger equation of the form $-\Delta u+V(x) u-\Delta\left(u^{2}\right) u=h(x, u), x \in R^{N}$. Under appropriate assumptions on $V(x)$ and $h(x, u)$, existence results of nontrivial solutions and high energy solutions are obtained by the dual-perturbation method.

\section{Introduction and Preliminaries}

In this paper we consider the quasilinear Schrödinger equation of the form

$$
-\Delta u+V(x) u-\Delta\left(u^{2}\right) u=h(x, u), \quad x \in R^{N},
$$

where $h \in C\left(R^{N} \times R, R\right)$ and $V \in C\left(R^{N}, R\right)$. Solutions of (1) are standing waves of the following quasilinear Schrödinger equation:

$$
\begin{aligned}
i \psi_{t}+ & \Delta \psi-V(x) \psi+k \Delta\left(\alpha\left(|\psi|^{2}\right)\right) \alpha^{\prime}\left(|\psi|^{2}\right) \psi \\
& +g(x, \psi)=0, \quad x \in R^{N},
\end{aligned}
$$

where $V(x)$ is a given potential, $k$ is a real constant, and $\alpha$ and $g$ are real functions. The quasilinear Schrödinger equations (2) are derived as models of several physical phenomena; for example, see [1-5]. Several methods can be used to solve (1). For instance, the existence of a positive ground state solution has been proved in $[6,7]$ by using a constrained minimization argument; the problem is transformed to a semilinear one in [8-11] by a change of variables (dual approach); Nehari method is used to get the existence results of ground state solutions in $[12,13]$.

Recently, some new methods have been applied to these equations. In [14], the authors prove that the critical points are $L^{\infty}$ functions by the Moser's iteration; then the existence of multibump type solutions is constructed for this class of quasilinear Schrödinger equations. In [15], by analysing the behavior of the solutions for subcritical case, the authors pass to the limit as the exponent approaches to the critical exponent in order to establish the existence of both onesign and nodal ground state solutions. Another new method which works for these equations is perturbations. In [16] 4Laplacian perturbations are led into these equations; then high energy solutions are obtained on bounded smooth domain.

In this paper, the perturbation, combined with dual approach, is applied to search the existence of nontrivial solution and sequence of high energy solutions of (1) on the whole space $R^{N}$. For simplicity we call this method the dualperturbation method.

We need the following several notations. Let $C_{c}^{\infty}\left(R^{N}\right)$ be the collection of smooth functions with compact support. Let

$$
H^{1}(R):=\left\{u \in L^{2}\left(R^{N}\right):|\nabla u| \in L^{2}\left(R^{N}\right)\right\},
$$

with the inner product

$$
\langle u, v\rangle_{H^{1}}=\int_{R^{N}}[\nabla u \cdot \nabla v+u v] d x
$$


and the norm

$$
\|u\|_{H^{1}}=\langle u, u\rangle_{H^{1}}^{1 / 2} .
$$

Let the following assumption $(V)$ hold:

$(V) V \in C\left(R^{N}, R\right)$ satisfies $\inf _{x \in R^{N}} V(x) \geq a_{0}>0$ and $\lim _{|x| \rightarrow \infty} V(x)=+\infty$.

Set

$$
E:=\left\{u \in H^{1}\left(R^{N}\right): \int_{\mathbb{R}^{N}} V(x) u^{2} d x<+\infty\right\}
$$

with the inner product

$$
\langle u, v\rangle_{E}=\int_{R^{N}}[\nabla u \cdot \nabla v+V(x) u v] d x
$$

and the norm

$$
\|u\|_{E}=\langle u, u\rangle_{E}^{1 / 2} .
$$

Then both $H^{1}\left(R^{N}\right)$ and $E$ are Hilbert spaces.

By the continuity of the embedding $E \hookrightarrow L^{s}\left(\mathbb{R}^{N}\right)$ for $s \in$ $\left[2,2^{*}\right]$ we know that, for each $s \in\left[2,2^{*}\right]$, there exists constant $a_{s}>0$ such that

$$
\|u\|_{s} \leq a_{s}\|u\|_{E}, \quad \forall u \in E,
$$

where $\|\cdot\|_{s}$ denotes the $L^{s}$-norm. In the following, we use $C$ or $C_{i}$ to denote various positive constants. Moreover, we need the following assumptions:

$\left(h_{1}\right)$ there exist $4<p<2\left(2^{*}\right)$ if $N \geq 3$ and $4<p<\infty$ if $N=2$ such that

$$
|h(x, s)| \leq C\left(1+|s|^{p-1}\right), \quad \forall s \in R,
$$

$\left(h_{2}\right) \lim _{s \rightarrow 0} h(x, s) / s=0$ uniformly in $x \in R^{N}$,

$\left(h_{3}\right)$ there exist $\mu>4$ and $r>0$ such that

$$
\begin{gathered}
c_{0}:=\inf _{x \in R^{N},|s|=r} H(x, s)>0, \\
\mu H(x, s) \leq h(x, s) s
\end{gathered}
$$

for all $x \in R^{N}$ and $|s| \geq r$, where $H(x, s)=\int_{0}^{s} h(x, t) d t$.

By Lemma 3.4 in [17] we know that, under the assumption $(V)$, the embedding $E \hookrightarrow L^{s}\left(R^{N}\right)$ is compact for each $2 \leq s<$ $2^{*}$.

Equation (1) is the Euler-Lagrange equation of the energy functional

$$
\begin{aligned}
J(u)= & \frac{1}{2} \int_{R^{N}}\left[\left(1+2 u^{2}\right)|\nabla u|^{2}+V(x) u^{2}\right] d x \\
& -\int_{R^{N}} H(x, u) d x,
\end{aligned}
$$

where $H(x, u)=\int_{0}^{u} h(x, t) d t$. Due to the presence of the term $\int_{R^{N}} u^{2}|\nabla u|^{2} d x, J(u)$ is not well defined in $E$. To overcome this difficulty, a dual approach is used in $[9,10]$. Following the idea from these papers, let $f$ be defined by

$$
f^{\prime}(t)=\frac{1}{\sqrt{1+2 f^{2}(t)}}
$$

on $[0,+\infty), f(0)=0$ and $f(-t)=-f(t)$ on $(-\infty, 0]$. Then $f$ has the following properties:

$\left(f_{1}\right) f$ is uniquely defined $C^{\infty}$ function and invertible;

$\left(f_{2}\right) 0<f^{\prime}(t) \leq 1$ for all $t \in R$;

$\left(f_{3}\right)|f(t)| \leq|t|$ for all $t \in R$;

$\left(f_{4}\right) \lim _{t \rightarrow 0} f(t) / t=1$;

$\left(f_{5}\right) \lim _{t \rightarrow+\infty} f(t) / \sqrt{t}=2^{1 / 4}, \lim _{t \rightarrow-\infty} f(t) / \sqrt{|t|}=-2^{1 / 4} ;$

$\left(f_{6}\right)(1 / 2) f(t) \leq t f^{\prime}(t) \leq f(t)$ for all $t \geq 0$ and $f(t) \leq$ $t f^{\prime}(t) \leq(1 / 2) f(t)$ for all $t \leq 0$;

$\left(f_{7}\right)|f(t)| \leq 2^{1 / 4} \sqrt{|t|}$ for all $t \in R$;

$\left(f_{8}\right)$ the function $f^{2}(t)$ is strictly convex;

$\left(f_{9}\right)$ there exists a positive $C$ such that

$$
|f(t)| \geq \begin{cases}C|t|, & |t| \leq 1, \\ C|t|^{1 / 2}, & |t| \geq 1\end{cases}
$$

$\left(f_{10}\right)$ there exist positive constants $C_{1}$ and $C_{2}$ such that

$$
|t| \leq C_{1}|f(t)|+C_{2}|f(t)|^{2}
$$

for all $t \in R$;

$\left(f_{11}\right)\left|f(t) f^{\prime}(t)\right| \leq 1 / \sqrt{2}$ for all $t \in R$;

$\left(f_{12}\right)$ for each $\xi>0$, there exists $C(\xi)>0$ such that $f^{2}(\xi t) \leq$ $C(\xi) f^{2}(t)$.

The properties $\left(f_{1}\right)-\left(f_{11}\right)$ have been proved in [8-11]. It suffices to prove $\left(f_{12}\right)$.

Indeed, by $\left(f_{1}\right),\left(f_{4}\right)$, and $\left(f_{5}\right)$, there exist $\delta>0$ and $M>0$ such that, for $|t| \leq \delta$,

$$
\frac{1}{2} t^{2} \leq f^{2}(t) \leq \frac{3}{2} t^{2}
$$

and for $|t| \geq M$,

$$
\frac{\sqrt{2}}{2}|t| \leq f^{2}(t) \leq \frac{3 \sqrt{2}}{2}|t| .
$$

Since there exists a $C_{0}>0$ such that $f^{2}(2 t) \leq C_{0} f^{2}(t)$ (see [10]), we can assume that $0<\xi<1$. For $|t| \leq \delta$, we have $|\xi t| \leq \delta$, and hence

$$
f^{2}(\xi t) \leq \frac{3}{2} \xi^{2} t^{2} \leq 3 \xi^{2} f^{2}(t) ;
$$

for $|t| \geq M / \xi>M$, one has $|\xi t| \geq M$, and hence

$$
f^{2}(\xi t) \leq \frac{3 \sqrt{2}}{2} \xi|t| \leq 3 \xi f^{2}(t) ;
$$


and for $\delta \leq|t| \leq M / \xi$, there exist $m(\xi)>0$ and $M(\xi)>0$ such that $f^{2}(\xi t) \leq M(\xi)$ and $f^{2}(t) \geq m(\xi)$. Then we have

$$
f^{2}(\xi t) \leq M(\xi) \leq \frac{M(\xi)}{m(\xi)} f^{2}(t) .
$$

Hence $f^{2}(\xi t) \leq C(\xi) f^{2}(t)$, where $C(\xi)=\max \left\{3 \xi^{2}, M(\xi) /\right.$ $m(\xi)\}$.

After the change of variable, $J(u)$ can be reduced to

$$
\begin{aligned}
I(v):= & J(f(v))=\frac{1}{2} \int_{R^{N}}\left(|\nabla v|^{2}+V(x) f^{2}(v)\right) d x \\
& -\int_{R^{N}} H(x, f(v)) d x .
\end{aligned}
$$

From $[8,9,11]$ we know that if $v \in E$ is a critical point of $I$, that is,

$$
\begin{aligned}
\left\langle I^{\prime}(v), \varphi\right\rangle= & \int_{R^{N}} \nabla v \nabla \varphi d x+\int_{R^{N}} V(x) f(v) f^{\prime}(v) \varphi d x \\
& -\int_{R^{N}} h(x, f(v)) f^{\prime}(v) \varphi d x=0
\end{aligned}
$$

for all $\varphi \in E$, then $u:=f(v)$ is a weak solution of (1). Particularly, if $v \in H^{1}\left(R^{N}\right) \cap C^{2}\left(R^{N}\right)$ is a critical point of $I$, then $u:=f(v)$ is a classical solution of (1).

A sequence $\left\{u_{n}\right\} \subset E$ is called a Cerami sequence of $J$ if $\left\{J\left(u_{n}\right)\right\}$ is bounded and $\left(1+\left\|u_{n}\right\|\right) J^{\prime}\left(u_{n}\right) \rightarrow 0$ in $E^{*}$. We say that $J$ satisfies the Cerami condition if every Cerami sequence possesses a convergent subsequence.

\section{Some Lemmas}

Consider the following perturbation functional $I_{\theta}$ defined by

$$
I_{\theta}(v)=I(v)+\frac{\theta}{2} \int_{R^{N}} V(x) v^{2} d x,
$$

where $\theta \in(0,1]$. We have the following lemmas.

Lemma 1. If assumptions $(V),\left(h_{1}\right)$, and $\left(h_{2}\right)$ hold, then the functional $I_{\theta}$ is well defined on $E$ and $I_{\theta} \in C^{1}(E, R)$.

Proof. By conditions $\left(h_{1}\right)$ and $\left(h_{2}\right)$, the properties $\left(f_{2}\right),\left(f_{3}\right)$, $\left(f_{7}\right)$, and $\left(f_{11}\right)$ imply that there exists $\delta>0$ such that

$$
\begin{aligned}
\left|h(x, f(v)) f^{\prime}(v)\right| & \leq|f(v)| f^{\prime}(v) \leq|v| \quad \text { for }|v|<\delta, \\
\left|h(x, f(v)) f^{\prime}(v)\right| & \leq C|f(v)|^{p-1} f^{\prime}(v) \\
& \leq C|f(v)|^{p-2} \leq C|v|^{(p-2) / 2} \text { for }|v| \geq \delta .
\end{aligned}
$$

Hence

$$
\begin{gathered}
\left|h(x, f(v)) f^{\prime}(v)\right| \leq C\left(|v|+|v|^{(p / 2)-1}\right), \\
|H(x, f(v))| \leq C\left(|v|^{2}+|v|^{p / 2}\right)
\end{gathered}
$$

for all $v \in R$. By (26) and the continuity of the embedding $E \hookrightarrow L^{s}\left(R^{N}\right)\left(s \in\left[2,2^{*}\right]\right)$,

$$
\int_{R^{N}} H(x, f(v)) d x<+\infty, \quad \forall v \in E .
$$

Hence $I_{\theta}$ is well defined in $E$.

Now, we prove that $I_{\theta} \in C^{1}(E, R)$. It suffices to prove that

$$
\begin{aligned}
& \Psi_{1}(v):=\int_{R^{N}} H(x, f(v)) d x \in C^{1}(E, R), \\
& \Psi_{2}(v):=\int_{R^{N}} V(x) f^{2}(v) d x \in C^{1}(E, R) .
\end{aligned}
$$

For any $v, \phi \in E$ and $0<|t|<1$, by the mean value theorem, (25) and $\left(f_{2}\right)-\left(f_{3}\right)$, we have

$$
\begin{aligned}
& \frac{|H(x, f(v+t \phi))-H(x, f(v))|}{|t|} \\
& \quad \leq \int_{0}^{1}\left|h(f(x, v+s t \phi)) f^{\prime}(v+s t \phi) \phi\right| d s \\
& \quad \leq C\left[|v||\phi|+|\phi|^{2}+|v|^{(p-2) / 2}|\phi|+|\phi|^{p / 2}\right], \\
& \frac{\left|V(x) f^{2}(v+t \phi)-V(x) f^{2}(v)\right|}{|t|} \\
& \quad \leq 2 \int_{0}^{1} V(x)\left|f(v+s t \phi) f^{\prime}(v+s t \phi) \phi\right| d s \\
& \quad \leq 2 V(x) \int_{0}^{1}|v+s t \phi||\phi| d s \\
& \leq 2 V(x)\left[|v||\phi|+|\phi|^{2}\right] .
\end{aligned}
$$

The Hölder inequality implies that

$$
\begin{gathered}
C\left[|v||\phi|+|\phi|^{2}+|v|^{(p-2) / 2}|\phi|+|\phi|^{p / 2}\right] \in L^{1}\left(R^{N}\right), \\
2 V(x)\left[|v||\phi|+|\phi|^{2}\right] \in L^{1}\left(R^{N}\right) .
\end{gathered}
$$

Hence, by the Lebesgue theorem, we have

$$
\begin{aligned}
\left\langle\Psi_{1}^{\prime}(v), \phi\right\rangle & =\int_{R^{N}} h(x, f(v)) f^{\prime}(v) \phi d x, \\
\left\langle\Psi_{2}^{\prime}(v), \phi\right\rangle & =2 \int_{R^{N}} V(x) f(v) f^{\prime}(v) \phi d x
\end{aligned}
$$

for all $\phi \in E$. Now, we show that $\Psi_{i}^{\prime}(\cdot): E \rightarrow E^{*}, i=1,2$, are continuous. Indeed, if $v_{n} \rightarrow v$ in $E$, then $v_{n} \rightarrow v$ in $L^{S}\left(R^{N}\right)$ for all $s \in\left[2,2^{*}\right]$.

On the space $L^{p_{1}}\left(R^{N}\right) \cap L^{p_{2}}\left(R^{N}\right)$, we define the norm

$$
\|v\|_{p_{1} \wedge p_{2}}=\|v\|_{p_{1}}+\|v\|_{p_{2}} .
$$

Then

$$
v_{n} \longrightarrow v \quad \text { in } L^{2}\left(R^{N}\right) \cap L^{(p / 2)-1}\left(R^{N}\right) .
$$


Moreover, on the space $L^{p_{1}}\left(R^{N}\right)+L^{p_{2}}\left(R^{N}\right)$, we define the norm

$$
\begin{aligned}
\|v\|_{p_{1} \vee p_{2}}=\inf \left\{\|u\|_{p_{1}}+\|w\|_{p_{2}}: v\right. \\
\left.\quad=u+w, u \in L^{p_{1}}\left(R^{N}\right), w \in L^{p_{2}}\left(R^{N}\right)\right\} .
\end{aligned}
$$

By (25), we have

$$
\begin{aligned}
\left|h(x, f(v)) f^{\prime}(v)\right| & \leq C\left(|v|+|v|^{(p / 2)-1}\right) \\
& \leq C\left(|v|^{2 / 2}+|v|^{q / r}\right),
\end{aligned}
$$

where $q=p / 2$ and $r=p /(p-2)$. Then Theorem A.4 in [18] implies

$$
\begin{array}{r}
h\left(x, f\left(v_{n}\right)\right) f^{\prime}\left(v_{n}\right)-h(x, f(v)) f^{\prime}(v) \longrightarrow 0 \\
\operatorname{in~} L^{2}\left(R^{N}\right)+L^{r}\left(R^{N}\right)
\end{array}
$$

as $n \rightarrow+\infty$. If $h\left(x, f\left(v_{n}\right)\right) f^{\prime}\left(v_{n}\right)-h(x, f(v)) f^{\prime}(v)=y_{n}+z_{n}$ with $y_{n} \in L^{2}\left(R^{N}\right)$ and $z_{n} \in L^{r}\left(R^{N}\right)$, one has

$$
\begin{aligned}
\mid \int_{R^{N}} & {\left[h\left(x, f\left(v_{n}\right)\right) f^{\prime}\left(v_{n}\right)-h(x, f(v)) f^{\prime}(v)\right] \phi d x \mid } \\
\leq & \int_{R^{N}}\left|y_{n}\right||\phi|+\left|z_{n}\right||\phi| d x \\
& \leq C\left(\left\|y_{n}\right\|_{2}+\left\|z_{n}\right\|_{r}\right)\|\phi\|_{E} .
\end{aligned}
$$

Hence

$$
\begin{aligned}
& \left|\int_{R^{N}}\left[h\left(x, f\left(v_{n}\right)\right) f^{\prime}\left(v_{n}\right)-h(x, f(v)) f^{\prime}(v)\right] \phi d x\right| \\
& \leq C\left\|h\left(x, f\left(v_{n}\right)\right) f^{\prime}\left(v_{n}\right)-h(x, f(v)) f^{\prime}(v)\right\|_{2 \vee r}\|\phi\|_{E},
\end{aligned}
$$

and hence

$$
\left\|\Psi_{1}^{\prime}\left(v_{n}\right)-\Psi_{1}^{\prime}(v)\right\| \longrightarrow 0
$$

as $n \rightarrow \infty$. Therefore, $\Psi_{1} \in C^{1}(E, R)$.

Define

$$
\begin{gathered}
L_{V}^{s}\left(R^{N}\right)=\left\{u: R^{N} \longrightarrow R: u\right. \text { is measurable } \\
\text { and } \left.\int_{R^{N}} V(x) u^{s} d x<\infty\right\}
\end{gathered}
$$

with the norm $\|u\|_{L_{V}^{s}}=\left(\int_{R^{N}} V(x) u^{s} d x\right)^{1 / s}$. On the space $L_{V}^{p_{1}}\left(R^{N}\right) \cap L_{V}^{p_{2}}\left(R^{N}\right)$, we define the norm

$$
\|v\|_{p_{1} \wedge p_{2}}=\|v\|_{L_{V}^{p_{1}}}+\|v\|_{L_{V}^{p_{2}}} .
$$

On the space $L_{V}^{p_{1}}\left(R^{N}\right)+L_{V}^{p_{2}}\left(R^{N}\right)$, we define the norm

$$
\begin{aligned}
\|v\|_{p_{1} \vee p_{2}}=\inf \left\{\|v\|_{p_{1} \vee p_{2}}=\|u\|_{L_{V}^{p_{1}}}+\|w\|_{L_{V}^{p_{2}}}:\right. \\
\left.v=u+w, u \in L_{V}^{p_{1}}\left(R^{N}\right), w \in L_{V}^{p_{2}}\left(R^{N}\right)\right\} .
\end{aligned}
$$

From $v_{n} \rightarrow v$ in $E$, one has $v_{n}, v \in L_{V}^{2}\left(R^{N}\right)$ and

$$
v_{n} \longrightarrow v \quad \text { in } L_{V}^{2}\left(R^{N}\right) \cap L_{V}^{2}\left(R^{N}\right)
$$

as $n \rightarrow \infty$. Since $\left|f(v) f^{\prime}(v)\right| \leq|v|$, by the following Lemma 2, we have

$$
f\left(v_{n}\right) f^{\prime}\left(v_{n}\right) \longrightarrow f(v) f^{\prime}(v) \quad \text { in } L_{V}^{2}\left(R^{N}\right)+L_{V}^{2}\left(R^{N}\right) .
$$

If $f\left(v_{n}\right) f^{\prime}\left(v_{n}\right)-f(v) f^{\prime}(v)=y_{n}+z_{n}$ with $y_{n} \in L_{V}^{2}\left(R^{N}\right)$ and $z_{n} \in L_{V}^{2}\left(R^{N}\right)$, one has

$$
\begin{aligned}
& \left|\int_{R^{N}} V(x)\left[f\left(v_{n}\right) f^{\prime}\left(v_{n}\right)-f(v) f^{\prime}(v)\right] \phi d x\right| \\
& \quad \leq \int_{R^{N}} V(x)\left|y_{n}\right||\phi|+V(x)\left|z_{n}\right||\phi| d x \\
& \quad \leq\left(\left\|y_{n}\right\|_{L_{V}^{2}}+\left\|z_{n}\right\|_{L_{V}^{2}}\right)\|\phi\|_{E} .
\end{aligned}
$$

Hence

$$
\begin{gathered}
\left|\int_{R^{N}} V(x)\left[f\left(v_{n}\right) f^{\prime}\left(v_{n}\right)-f(v) f^{\prime}(v)\right] \phi d x\right| \\
\leq\left\|f\left(v_{n}\right) f^{\prime}\left(v_{n}\right)-f(v) f^{\prime}(v)\right\|_{2 \vee 2}\|\phi\|_{E},
\end{gathered}
$$

and hence

$$
\left\|\Psi_{2}^{\prime}\left(v_{n}\right)-\Psi_{2}^{\prime}(v)\right\| \longrightarrow 0
$$

as $n \rightarrow \infty$. Therefore, $\Psi_{2} \in C^{1}(E, R)$. This completes the proof.

Lemma 2. Assume that $1 \leq p, q, r, s<+\infty, g \in C\left(R^{N} \times R\right)$ and

$$
|g(x, v)| \leq C\left(|v|^{p / r}+|v|^{q / s}\right) .
$$

Then, for every $v \in L_{V}^{p}\left(R^{N}\right) \cap L_{V}^{q}\left(R^{N}\right), g(\cdot, v) \in L_{V}^{r}\left(R^{N}\right)+$ $L_{V}^{s}\left(R^{N}\right)$, and the operator

$$
\begin{aligned}
A: L_{V}^{p} & \left(R^{N}\right) \cap L_{V}^{q}\left(R^{N}\right) \\
& \longrightarrow L_{V}^{r}\left(R^{N}\right)+L_{V}^{s}\left(R^{N}\right): v \longmapsto g(x, v)
\end{aligned}
$$

is continuous.

Proof. Let $\eta(s)$ be a smooth cut-off function such that $\eta(s)=1$ for $|s| \leq 1$ and $\eta(s)=0$ for $|s| \geq 2$. Define

$$
\begin{gathered}
g_{1}(x, v):=\eta(v) g(x, v), \\
g_{2}(x, v):=(1-\eta(v)) g(x, v) .
\end{gathered}
$$

We can assume that $p / r \leq q / s$. Hence

$$
\left|g_{1}(x, v)\right| \leq C|v|^{p / r}, \quad\left|g_{2}(x, v)\right| \leq C|v|^{q / s}
$$


for all $(x, v) \in R^{N} \times R$. Assume $v_{n} \rightarrow v$ in $L_{V}^{p}\left(R^{N}\right) \cap L_{V}^{q}\left(R^{N}\right)$. Then $v_{n} \rightarrow v$ in $L_{V}^{p}\left(R^{N}\right)$ and $g\left(\cdot, v_{n}\right) \rightarrow g(\cdot, v)$ in $L_{V}^{r}\left(R^{N}\right)$. As in the proof of Lemma A.1 in [18], there exists a subsequence $\left\{w_{n}\right\}$ of $\left\{v_{n}\right\}$ and $\alpha \in L_{V}^{p}\left(R^{N}\right)$ such that $w_{n}(x) \rightarrow v(x)$ and $|v(x)|,\left|w_{n}(x)\right| \leq \alpha(x)$ for a.e. $x \in R^{N}$. Hence, from (51), one has

$$
\left|g_{1}\left(x, w_{n}\right)-g_{1}(x, v)\right|^{r} \leq 2^{r} C|\alpha(x)|^{p}
$$

a.e. on $R^{N}$. It follows from the Lebesgue theorem that $g_{1}\left(\cdot, w_{n}\right) \rightarrow g_{1}(\cdot, v)$ in $L_{V}^{r}\left(R^{N}\right)$. Consequently, $g_{1}\left(\cdot, v_{n}\right) \rightarrow$ $g_{1}(\cdot, v)$ in $L_{V}^{r}\left(R^{N}\right)$. Similarly, we can prove $g_{2}\left(\cdot, v_{n}\right) \rightarrow$ $g_{2}(\cdot, v)$ in $L_{V}^{s}$. Since

$$
\begin{aligned}
\left\|g\left(\cdot, v_{n}\right)-g(\cdot, v)\right\|_{r \vee s} \leq & \left\|g_{1}\left(\cdot, v_{n}\right)-g_{1}(\cdot, v)\right\|_{L_{V}^{r}} \\
& +\left\|g_{2}\left(\cdot, v_{n}\right)-g_{2}(\cdot, v)\right\|_{L_{V}^{s}}
\end{aligned}
$$

it follows that $g\left(\cdot, v_{n}\right) \rightarrow g(\cdot, v)$ in $L_{V}^{r}+L_{V}^{s}$. This completes the proof.

Lemma 3. Let $(V),\left(h_{1}\right)$, and $\left(h_{2}\right)$ hold. Then every bounded sequence $\left\{v_{n}\right\} \subset E$ with $I_{\theta}^{\prime}\left(v_{n}\right) \rightarrow 0$ possesses a convergent subsequence.

Proof. Since $\left\{v_{n}\right\} \subset E$ is bounded, then, by the compactness of the embedding $E \hookrightarrow L^{s}\left(R^{N}\right)\left(2 \leq s<2^{*}\right)$, passing to a subsequence, one has $v_{n} \rightarrow v$ in $E, v_{n} \rightarrow v$ in $L^{s}\left(R^{N}\right)$ for all $2 \leq s<2^{*}$, and $v_{n}(x) \rightarrow v(x)$ for a.e. $x \in R^{N}$. By (25)

$$
\begin{array}{rl}
\mid \int_{R^{N}} & h\left(x, f\left(v_{n}\right)\right) f^{\prime}\left(v_{n}\right)\left(v-v_{n}\right) d x \mid \\
\quad & \int_{R^{N}} C\left(\left|v_{n}\right|+\left|v_{n}\right|^{(p / 2)-1}\right)\left|v_{n}-v\right| d x \\
& \leq C\left(\left\|v_{n}\right\|_{2}\left\|v_{n}-v\right\|_{2}+\left\|v_{n}\right\|_{p / 2}^{(p / 2)-1}\left\|v_{n}-v\right\|_{p / 2}\right) \\
& \leq C\left(\left\|v_{n}\right\|_{E}\left\|v_{n}-v\right\|_{2}+\left\|v_{n}\right\|_{E}^{(p / 2)-1}\left\|v_{n}-v\right\|_{p / 2}\right) \longrightarrow 0
\end{array}
$$

as $n \rightarrow \infty$. Similarly, $\int_{R^{N}} h(x, f(v)) f^{\prime}(v)\left(v-v_{n}\right) d x \rightarrow 0$ as $n \rightarrow \infty$. Hence, by the property of $\left(f_{8}\right)$, we have

$$
\begin{aligned}
& \left\langle I_{\theta}^{\prime}\left(v_{n}\right)-I_{\theta}^{\prime}(v), v_{n}-v\right\rangle \\
& =\int_{R^{N}}\left|\nabla\left(v_{n}-v\right)\right|^{2} d x+\theta \int_{R^{N}} V(x)\left|v_{n}-v\right|^{2} d x \\
& \quad+\int_{R^{N}} V(x)\left[f\left(v_{n}\right) f^{\prime}\left(v_{n}\right)-f(v) f^{\prime}(v)\right] \\
& \quad \times\left(v_{n}-v\right) d x \\
& \quad-\int_{R^{N}}\left[h\left(x, f\left(v_{n}\right)\right) f^{\prime}\left(v_{n}\right)\right. \\
& \left.\quad-h(x, f(v)) f^{\prime}(v)\right]\left(v_{n}-v\right) d x \\
& \geq \theta\left\|v_{n}-v\right\|_{E}^{2}-o_{n}(1),
\end{aligned}
$$

where $o_{n}(1) \rightarrow 0$ as $n \rightarrow \infty$. This shows that $\left\|v_{n}-v\right\|_{E}^{2} \rightarrow 0$ as $n \rightarrow \infty$. This completes the proof.
The following Lemma 4 has been proved in [10] (see Proposition 2.1(3) in [10]).

Lemma 4. If $v_{n}(x) \rightarrow v(x)$ a.e. in $R^{N}$ and $\lim _{n \rightarrow \infty} \int_{R^{N}} V(x) f^{2}\left(v_{n}\right) d x=\int_{R^{N}} V(x) f^{2}(v) d x$, then $\int_{R^{N}} V(x) f^{2}\left(v_{n}-v\right) d x \rightarrow 0$ as $n \rightarrow \infty$.

\section{Main Results}

Theorem 5. Assume conditions $(V),\left(h_{1}\right)-\left(h_{3}\right)$ hold. Let $\left\{\theta_{n}\right\} \subset$ $(0,1]$ be such that $\theta_{n} \rightarrow 0$. Let $v_{n} \in E$ be a critical point of $I_{\theta_{n}}$ with $I_{\theta_{n}}\left(v_{n}\right) \leq c$ for some constant $c$ independent of $n$. Then, $u p$ to subsequence, one has $v_{n} \rightarrow v$ in $E, I_{\theta_{n}}\left(v_{n}\right) \rightarrow I(v)$ and $v$ is a critical point of $I$.

Proof. By $\left(h_{2}\right)$, for $0<\varepsilon_{0}<(1 / 4)(1 / 2-1 / \mu) a_{0}$, there exists $\delta_{0}>0$ such that

$$
\left|\frac{1}{\mu} \operatorname{sh}(x, s)-H(x, s)\right| \leq \varepsilon_{0} s^{2}, \quad \forall s \in\left[-\delta_{0}, \delta_{0}\right] .
$$

By $\left(h_{1}\right)$, for $\delta_{0} \leq|s| \leq r$ ( $r$ is the constant appearing in condition $\left.\left(h_{3}\right)\right)$, we have

$$
\left|\frac{1}{\mu} \operatorname{sh}(x, s)-H(x, s)\right| \leq 2 C\left(\frac{1}{\delta_{0}^{2}}+r^{p-2}\right) s^{2},
$$

where $C$ is the constant appearing in condition $\left(h_{1}\right)$. Hence

$$
\begin{array}{r}
\left|\frac{1}{\mu} s h(x, s)-H(x, s)\right| \leq \varepsilon_{0} s^{2}+2 C\left(\frac{1}{\delta_{0}^{2}}+r^{p-2}\right) s^{2}, \\
\forall s \in[-r, r] .
\end{array}
$$

Since $\lim _{|x| \rightarrow \infty} V(x)=+\infty$, there exists $\rho_{0}>0$ such that

$$
\frac{1}{4}\left(\frac{1}{2}-\frac{1}{\mu}\right) V(x)>2 C\left(\frac{1}{\delta_{0}^{2}}+r^{p-2}\right)
$$

for all $|x| \geq \rho_{0}$. Hence

$$
\begin{gathered}
\left(\frac{1}{2}-\frac{1}{\mu}\right) \int_{R^{N}} V(x) f^{2}(v) d x \\
+\int_{\{x:|f(v)| \leq r\}}\left[\frac{1}{\mu} f(v) h(x, f(v))\right. \\
-H(x, f(v))] d x \\
\geq \frac{1}{2}\left(\frac{1}{2}-\frac{1}{\mu}\right) \int_{R^{N}} V(x) f^{2}(v) d x \\
-2 C\left(\frac{1}{\delta_{0}^{2}}+r^{p-2}\right) r^{2}\left|B_{\rho_{0}}\right| .
\end{gathered}
$$


6

Abstract and Applied Analysis

Since $v_{n}$ is a critical point of $I_{\theta_{n}}$,

$$
\begin{aligned}
\left\langle I_{\theta_{n}}^{\prime}\left(v_{n}\right), \phi\right\rangle= & \int_{R^{N}} \nabla v_{n} \nabla \phi d x \\
& +\int_{R^{N}} V(x) f\left(v_{n}\right) f^{\prime}\left(v_{n}\right) \phi d x \\
& +\theta_{n} \int_{R^{N}} V(x) v_{n} \phi d x \\
& -\int_{R^{N}} h\left(x, f\left(v_{n}\right)\right) f^{\prime}\left(v_{n}\right) \phi d x=0
\end{aligned}
$$

for all $\phi \in E$. Consequently, taking $\phi=f\left(v_{n}\right) / f^{\prime}\left(v_{n}\right) \in E$, by $\left(h_{3}\right)$ and $\left(f_{6}\right)$ we have

$$
\begin{aligned}
& c \geq I_{\theta_{n}}\left(v_{n}\right)=I_{\theta_{n}}\left(v_{n}\right)-\frac{1}{\mu}\left\langle I_{\theta_{n}}^{\prime}\left(v_{n}\right), \frac{f\left(v_{n}\right)}{f^{\prime}\left(v_{n}\right)}\right\rangle \\
& =\int_{R^{N}}\left[\frac{1}{2}-\frac{1}{\mu}\left(1+\frac{2 f^{2}\left(v_{n}\right)}{1+2 f^{2}\left(v_{n}\right)}\right)\right]\left|\nabla v_{n}\right|^{2} d x \\
& +\left(\frac{1}{2}-\frac{1}{\mu}\right) \int_{R^{N}} V(x) f^{2}\left(v_{n}\right) d x \\
& +\frac{\theta_{n}}{2} \int_{R^{N}} V(x) v_{n}^{2} d x \\
& -\frac{\theta_{n}}{\mu} \int_{R^{N}} V(x) \frac{v_{n} f\left(v_{n}\right)}{f^{\prime}\left(v_{n}\right)} d x \\
& +\int_{R^{N}}\left[\frac{1}{\mu} h\left(x, f\left(v_{n}\right)\right) f\left(v_{n}\right)-H\left(x, f\left(v_{n}\right)\right)\right] d x \\
& \geq\left(\frac{1}{2}-\frac{2}{\mu}\right) \int_{R^{N}}\left|\nabla v_{n}\right|^{2} d x+\left(\frac{1}{2}-\frac{1}{\mu}\right) \int_{R^{N}} V(x) f^{2}\left(v_{n}\right) d x \\
& +\left(\frac{1}{2}-\frac{2}{\mu}\right) \theta_{n} \int_{R^{N}} V(x) v_{n}^{2} d x \\
& +\int_{\left\{x:\left|f\left(v_{n}\right)\right| \leq r\right\}}\left[\frac{1}{\mu} h\left(x, f\left(v_{n}\right)\right) f\left(v_{n}\right)\right. \\
& \left.-H\left(x, f\left(v_{n}\right)\right)\right] d x \\
& \geq\left(\frac{1}{2}-\frac{2}{\mu}\right) \int_{R^{N}}\left|\nabla v_{n}\right|^{2} d x \\
& +\frac{1}{2}\left(\frac{1}{2}-\frac{1}{\mu}\right) \int_{R^{N}} V(x) f^{2}\left(v_{n}\right) d x \\
& +\left(\frac{1}{2}-\frac{2}{\mu}\right) \theta_{n} \int_{R^{N}} V(x) v_{n}^{2} d x \\
& -2 C\left(\frac{1}{\delta_{0}^{2}}+r^{p-2}\right) r^{2}\left|B_{\rho_{0}}\right|
\end{aligned}
$$

$$
\begin{aligned}
\geq & \left(\frac{1}{2}-\frac{2}{\mu}\right) \int_{R^{N}}\left|\nabla v_{n}\right|^{2} d x \\
& +\left(\frac{1}{4}-\frac{1}{2 \mu}\right) \int_{R^{N}} V(x) f^{2}\left(v_{n}\right) d x \\
& +\left(\frac{1}{2}-\frac{2}{\mu}\right) \theta_{n} \int_{R^{N}} V(x) v_{n}^{2} d x-C_{1},
\end{aligned}
$$

and hence

$$
\begin{gathered}
\int_{R^{N}}\left|\nabla v_{n}\right|^{2} d x+\int_{R^{N}} V(x) f^{2}\left(v_{n}\right) d x \\
+\theta_{n} \int_{R^{N}} V(x) v_{n}^{2} d x \leq C
\end{gathered}
$$

for some constant $C$ independent of $n$. By the boundedness of $\int_{R^{N}}\left|\nabla v_{n}\right|^{2} d x$, there exists $C_{2}>0$ such that

$$
\begin{aligned}
2 \int_{\mathbb{R}^{N}} & f^{2}\left(v_{n}\right)\left|\nabla f\left(v_{n}\right)\right|^{2} d x \\
& \leq \int_{\mathbb{R}^{N}}\left[1+2 f^{2}\left(v_{n}\right)\right]\left|\nabla f\left(v_{n}\right)\right|^{2} d x \\
& =\int_{\mathbb{R}^{N}}\left|\nabla v_{n}\right|^{2} d x \leq C_{2}
\end{aligned}
$$

for all $n$. Hence, by the Sobolev embedding theorem, one has

$$
\left\|f\left(v_{n}\right)\right\|_{2\left(2^{*}\right)}^{4}=\left\|f^{2}\left(v_{n}\right)\right\|_{2^{*}}^{2} \leq C_{3}\left\|\nabla f^{2}\left(v_{n}\right)\right\|_{2}^{2} \leq C .
$$

Next, we prove that $f\left(v_{n}\right) \in L^{\infty}\left(R^{N}\right)$ and $\left\|f\left(v_{n}\right)\right\|_{L^{\infty}} \leq$ $C$, where the positive constant $C$ is independent of $n$. Setting $T>2, r>0$, define $\widetilde{v}_{n}^{T}=b\left(v_{n}\right)$, where $b: R \rightarrow R$ is a smooth function satisfying $b(s)=s$ for $|s| \leq T-1, b(-s)=-b(s)$; $b^{\prime}(s)=0$ for $s \geq T$, and $b^{\prime}(s)$ is decreasing in $[T-1, T]$.

This means that $\widetilde{v}_{n}^{T}=v_{n}$, for $\left|v_{n}\right| \leq T-1 ;\left|\widetilde{v}_{n}^{T}\right|=\left|b\left(v_{n}\right)\right| \leq$ $\left|v_{n}\right|$, for $T-1 \leq\left|v_{n}\right| \leq T ;\left|\widetilde{v}_{n}^{T}\right|=C_{T}>0$, for $\left|v_{n}\right| \geq T$, where $T-1 \leq C_{T} \leq T$.

Let $\phi=\left(f\left(v_{n}\right) / f^{\prime}\left(v_{n}\right)\right)\left|f\left(\widetilde{v}_{n}^{T}\right)\right|^{2 r}$; then $\phi \in E$. By $(61)$ $\left\langle I^{\prime}\left(v_{n}\right), \phi\right\rangle=0$. Hence

$$
\begin{aligned}
I_{1}+I_{2} & +I_{3}+I_{4}+I_{5} \\
& =\int_{\mathbb{R}^{N}} h\left(x, f\left(v_{n}\right)\right) f\left(v_{n}\right)\left|f\left(\widetilde{v}_{n}^{T}\right)\right|^{2 r} d x,
\end{aligned}
$$


where

$$
\begin{aligned}
& I_{5}:=\int_{R^{N}} V(x) f^{2}\left(v_{n}\right)\left|f\left(\tilde{v}_{n}^{T}\right)\right|^{2 r} d x \\
& +\theta_{n} \int_{R^{N}} V(x) \frac{v_{n} f\left(v_{n}\right)}{f^{\prime}\left(v_{n}\right)}\left|f\left(\widetilde{v}_{n}^{T}\right)\right|^{2 r} d x \\
& \geq \int_{R^{N}} V(x) f^{2}\left(v_{n}\right)\left|f\left(\widetilde{v}_{n}^{T}\right)\right|^{2 r} d x, \\
& I_{1}:=\int_{\left\{x:\left|v_{n}\right| \geq T\right\}}\left[1+\frac{2 f^{2}\left(v_{n}\right)}{1+2 f^{2}\left(v_{n}\right)}\right]\left|f\left(\widetilde{v}_{n}^{T}\right)\right|^{2 r}\left|\nabla v_{n}\right|^{2} d x \\
& \geq \int_{\left\{x:\left|v_{n}\right| \geq T\right\}}\left|f\left(\widetilde{v}_{n}^{T}\right)\right|^{2 r}\left|\nabla v_{n}\right|^{2} d x \\
& =\int_{\left\{x:\left|v_{n}\right| \geq T\right\}}\left[1+2 f^{2}\left(v_{n}\right)\right] \\
& \times\left|\nabla f\left(v_{n}\right)\right|^{2}\left|f\left(\widetilde{v}_{n}^{T}\right)\right|^{2 r} d x \\
& \geq 2 \int_{\left\{x:\left|v_{n}\right| \geq T\right\}} f^{2}\left(v_{n}\right)\left|\nabla f\left(v_{n}\right)\right|^{2}\left|f\left(\widetilde{v}_{n}^{T}\right)\right|^{2 r} d x \\
& =\frac{1}{2} \int_{\left\{x:\left|v_{n}\right| \geq T\right\}}\left|\nabla\left[f^{2}\left(v_{n}\right) f^{r}\left(\widetilde{v}_{n}^{T}\right)\right]\right|^{2} d x, \\
& I_{2}:=\int_{\left\{x:\left|v_{n}\right| \leq T-1\right\}}\left[2 r+1+\frac{2 f^{2}\left(v_{n}\right)}{1+2 f^{2}\left(v_{n}\right)}\right] \\
& \times\left|f\left(\widetilde{v}_{n}^{T}\right)\right|^{2 r}\left|\nabla v_{n}\right|^{2} d x \\
& \geq \int_{\left\{x:\left|v_{n}\right| \leq T-1\right\}} \frac{2 f^{2}\left(v_{n}\right)}{1+2 f^{2}\left(v_{n}\right)}\left|f\left(\widetilde{v}_{n}^{T}\right)\right|^{2 r}\left|\nabla v_{n}\right|^{2} d x \\
& \geq \int_{\left\{x:\left|v_{n}\right| \leq T-1\right\}}\left|f\left(v_{n}\right)\right|^{2 r+2}\left|\nabla f\left(v_{n}\right)\right|^{2} d x \\
& =\frac{1}{(r+2)^{2}} \int_{\left\{x:\left|v_{n}\right| \leq T-1\right\}}\left|\nabla f^{r+2}\left(v_{n}\right)\right|^{2} d x \\
& =\frac{1}{(r+2)^{2}} \int_{\left\{x:\left|v_{n}\right| \leq T-1\right\}}\left|\nabla\left[f^{2}\left(v_{n}\right) f^{r}\left(\widetilde{v}_{n}^{T}\right)\right]\right|^{2} d x \text {, } \\
& I_{3}:=\int_{\left\{x: T-1 \leq v_{n} \mid \leq T\right\}}\left[1+\frac{2 f^{2}\left(v_{n}\right)}{1+2 f^{2}\left(v_{n}\right)}\right] f^{2 r}\left(\widetilde{v}_{n}^{T}\right)\left|\nabla v_{n}\right|^{2} d x \\
& \geq \int_{\left\{x: T-1 \leq\left|v_{n}\right| \leq T\right\}} \frac{2 f^{2}\left(v_{n}\right)}{1+2 f^{2}\left(v_{n}\right)} f^{2 r}\left(\widetilde{v}_{n}^{T}\right)\left|\nabla v_{n}\right|^{2} d x \\
& =\frac{1}{2} \int_{\left\{x: T-1 \leq\left|v_{n}\right| \leq T\right\}}\left[f^{r}\left(\widetilde{v}_{n}^{T}\right) \nabla f^{2}\left(v_{n}\right)\right]^{2} d x \text {, } \\
& I_{4}:=2 r \int_{\left\{x: T-1 \leq\left|v_{n}\right| \leq T\right\}} f^{2 r-1}\left(\widetilde{v}_{n}^{T}\right) f^{\prime}\left(\widetilde{v}_{n}^{T}\right) \\
& \times b^{\prime}\left(v_{n}\right) \frac{f\left(v_{n}\right)}{f^{\prime}\left(v_{n}\right)}\left|\nabla v_{n}\right|^{2} d x .
\end{aligned}
$$

For $T-1 \leq\left|v_{n}\right| \leq T$, $\left|\widetilde{v}_{n}^{T}\right|=\left|b\left(v_{n}\right)\right| \leq\left|v_{n}\right|$. By the properties of $f$ and $b$, the mean value theorem implies

$$
\begin{aligned}
\left|f\left(b\left(v_{n}\right)\right)\right| & \geq f^{\prime}\left(b\left(v_{n}\right)\right) b^{\prime}\left(v_{n}\right)\left|v_{n}\right| \\
& \geq \frac{1}{\sqrt{2}} f^{\prime}\left(b\left(v_{n}\right)\right) b^{\prime}\left(v_{n}\right) f^{2}\left(v_{n}\right) .
\end{aligned}
$$

Hence

$$
\begin{aligned}
& I_{4}=2 r \int_{\left\{x: T-1 \leq\left|v_{n}\right| \leq T\right\}} f^{2 r-1}\left(\widetilde{v}_{n}^{T}\right) f^{\prime}\left(\widetilde{v}_{n}^{T}\right) \\
& \times b^{\prime}\left(v_{n}\right) \frac{f\left(v_{n}\right)}{f^{\prime}\left(v_{n}\right)}\left|\nabla v_{n}\right|^{2} d x \\
& =2 r \int_{\left\{x: T-1 \leq\left|v_{n}\right| \leq T\right\}} f^{2 r-1}\left(b\left(v_{n}\right)\right) f^{\prime}\left(b\left(v_{n}\right)\right) \\
& \times b^{\prime}\left(v_{n}\right) f\left(v_{n}\right) \\
& \times \sqrt{1+2 f^{2}\left(v_{n}\right)}\left|\nabla v_{n}\right|^{2} d x \\
& \geq 2 r \int_{\left\{x: T-1 \leq\left|v_{n}\right| \leq T\right\}}\left[f^{r-1}\left(b\left(v_{n}\right)\right) f^{\prime}\left(b\left(v_{n}\right)\right) b^{\prime}\left(v_{n}\right)\right]^{2} \\
& \times f\left(v_{n}\right) v_{n} \sqrt{2 f^{2}\left(v_{n}\right)}\left|\nabla v_{n}\right|^{2} d x \\
& \geq 2 r \int_{\left\{x: T-1 \leq\left|v_{n}\right| \leq T\right\}}\left[f^{r-1}\left(b\left(v_{n}\right)\right) f^{\prime}\left(b\left(v_{n}\right)\right) b^{\prime}\left(v_{n}\right)\right]^{2} \\
& \times f^{4}\left(v_{n}\right)\left|\nabla v_{n}\right|^{2} d x \\
& =2 r \int_{\left\{x: T-1 \leq\left|v_{n}\right| \leq T\right\}} f^{4}\left(v_{n}\right) \\
& \times\left[f^{r-1}\left(b\left(v_{n}\right)\right) \nabla f\left(b\left(v_{n}\right)\right)\right]^{2} d x \\
& =\frac{2}{r} \int_{\left\{x: T-1 \leq\left|v_{n}\right| \leq T\right\}}\left[f^{2}\left(v_{n}\right) \nabla f^{r}\left(\widetilde{v}_{n}^{T}\right)\right]^{2} d x .
\end{aligned}
$$

Consequently,

$$
\begin{aligned}
I_{3}+I_{4}= & \frac{1}{2} \int_{\left\{x: T-1 \leq\left|v_{n}\right| \leq T\right\}}\left[f^{r}\left(\widetilde{v}_{n}^{T}\right) \nabla f^{2}\left(v_{n}\right)\right]^{2} d x \\
& +\frac{2}{r} \int_{\left\{x: T-1 \leq v_{n} \mid \leq T\right\}}\left[f^{2}\left(v_{n}\right) \nabla f^{r}\left(\widetilde{v}_{n}^{T}\right)\right]^{2} d x \\
\geq & \frac{1}{(r+2)^{2}} \int_{\left\{x: T-1 \leq v_{n} \mid \leq T\right\}} 2\left[f^{r}\left(\widetilde{v}_{n}^{T}\right) \nabla f^{2}\left(v_{n}\right)\right]^{2} \\
& +2\left[f^{2}\left(v_{n}\right) \nabla f^{r}\left(\widetilde{v}_{n}^{T}\right)\right]^{2} d x \\
\geq & \frac{1}{(r+2)^{2}} \int_{\left\{x: T-1 \leq\left|v_{n}\right| \leq T\right\}}\left|\nabla\left[f^{2}\left(v_{n}\right) f^{r}\left(\widetilde{v}_{n}^{T}\right)\right]\right|^{2} d x .
\end{aligned}
$$


Combining (67) and (68), we have

$$
I_{1}+I_{2}+I_{3}+I_{4} \geq \frac{1}{(r+2)^{2}} \int_{\mathbb{R}^{N}}\left|\nabla\left[f^{2}\left(v_{n}\right) f^{r}\left(\widetilde{v}_{n}^{T}\right)\right]\right|^{2} d x
$$

For any $\varepsilon>0$, by $\left(h_{1}\right)$ and $\left(h_{2}\right)$, there exists $C(\varepsilon)>0$ such that

$$
|h(x, s)| \leq \varepsilon|s|+C(\varepsilon)|s|^{p-1}
$$

Combining (66), (72), and (73), one has

$$
\begin{aligned}
& \frac{1}{(r+2)^{2}} \int_{R^{N}}\left|\nabla\left[f^{2}\left(v_{n}\right) f^{r}\left(\widetilde{v}_{n}^{T}\right)\right]\right|^{2} d x \\
& \leq C(\varepsilon) \int_{R^{N}}\left|f\left(v_{n}\right)\right|^{p}\left|f\left(\widetilde{v}_{n}^{T}\right)\right|^{2 r} d x .
\end{aligned}
$$

By the Hölder inequality and (65),

$$
\begin{aligned}
& \int_{R^{N}}\left|f\left(v_{n}\right)\right|^{p}\left|f\left(\tilde{v}_{n}^{T}\right)\right|^{2 r} d x \\
& =\int_{R^{N}}\left|f\left(v_{n}\right)\right|^{p-4}\left|f\left(\widetilde{v}_{n}^{T}\right)\right|^{2 r} f^{4}\left(v_{n}\right) d x \\
& \leq\left(\int_{R^{N}}\left|f\left(v_{n}\right)\right|^{(p-4) \cdot(4 N /(p-4)(N-2))} d x\right)^{(p-4)(N-2) / 4 N} \\
& \cdot\left(\int _ { R ^ { N } } \left[\left|f\left(\widetilde{v}_{n}^{T}\right)\right|^{2 r}\right.\right. \\
& \left.=\left(\int_{R^{N}}\left|f\left(v_{n}\right)\right|^{22^{*}} d x\right)^{((p-4)(N-2)) / 4 N} d x\right)^{(4 N-(p-4)(N-2)) / 4 N} \\
& \quad \cdot\left(\int _ { R ^ { N } } \left[\left|f\left(\tilde{v}_{n}^{T}\right)\right|^{r}\right.\right. \\
& \left.\quad \times f^{2}\left(v_{n}^{2}\left(v_{n}\right)\right]^{8 N /(4 N-(4 N-(p-4)(N-2))} d x\right)^{(4 N-(p-4)(N-2)) / 4 N} \\
& \leq C\left(\int _ { R ^ { N } } \left[\left|f\left(\widetilde{v}_{n}^{T}\right)\right|^{r}\right.\right.
\end{aligned}
$$

Moreover,

$$
\begin{aligned}
& \frac{1}{(r+2)^{2}} \int_{R^{N}}\left|\nabla\left[f^{2}\left(v_{n}\right) f^{r}\left(\widetilde{v}_{n}^{T}\right)\right]\right|^{2} d x \\
& \quad \geq \frac{C}{(r+2)^{2}}\left(\int_{R^{N}}\left[f^{2}\left(v_{n}\right)\left|f\left(\widetilde{v}_{n}^{T}\right)\right|^{r}\right]^{2^{*}} d x\right)^{2 / 2^{*}} .
\end{aligned}
$$

Hence

$$
\begin{aligned}
& \left(\int_{R^{N}}\left[f^{2}\left(v_{n}\right)\left|f\left(\widetilde{v}_{n}^{T}\right)\right|^{r}\right]^{2^{*}} d x\right)^{2 / 2^{*}} \\
& \leq C(r+2)^{2} \\
& \quad \times\left(\int _ { R ^ { N } } \left[\left|f\left(\widetilde{v}_{n}^{T}\right)\right|^{r}\right.\right. \\
& \left.\left.\quad \times f^{2}\left(v_{n}\right)\right]^{8 N /(4 N-(p-4)(N-2))} d x\right)^{(4 N-(p-4)(N-2)) / 4 N} .
\end{aligned}
$$

Since $4<p<2\left(2^{*}\right), d=2^{*} /(8 N /(4 N-(p-4)(N-2)))=$ $2^{*} / 2-p / 4+1>1$. Set $q=8 N /(4 N-(p-4)(N-2))$. Then

$$
\begin{aligned}
\left(\int_{R^{N}}[\right. & \left.\left.f^{2}\left(v_{n}\right)\left|f\left(\widetilde{v}_{n}^{T}\right)\right|^{r}\right]^{q d} d x\right)^{1 / q d(r+2)} \\
\leq & {\left[C(r+2)^{2}\right]^{1 / 2(r+2)} } \\
& \times\left(\int_{R^{N}}\left[\left|f\left(\widetilde{v}_{n}^{T}\right)\right|^{r} f^{2}\left(v_{n}\right)\right]^{q} d x\right)^{1 / q(r+2)} .
\end{aligned}
$$

Take $r=r_{0}$ such that $\left(2+r_{0}\right) q=2\left(2^{*}\right)$. Since $\left|\widetilde{v}_{n}^{T}\right|=\left|b\left(v_{n}\right)\right| \leq$ $\left|v_{n}\right|,\left|f\left(\widetilde{v}_{n}^{T}\right)\right| \leq\left|f\left(v_{n}\right)\right|$. Hence, from (65), we have

$$
\int_{R^{N}}\left[\left|f\left(\widetilde{v}_{n}^{T}\right)\right|^{r_{0}} f^{2}\left(v_{n}\right)\right]^{q} d x \leq \int_{R^{N}}\left|f\left(v_{n}\right)\right|^{\left(2+r_{0}\right) q} d x<C .
$$

Since $f\left(\widetilde{v}_{n}^{T}\right) \rightarrow f\left(v_{n}\right)$ as $T \rightarrow+\infty$, taking $T \rightarrow+\infty$ in $(78)$ with $r=r_{0}$, we have

$$
\begin{aligned}
\left(\int_{R^{N}}\left|f\left(v_{n}\right)\right|^{\left(2+r_{0}\right) q d} d x\right)^{1 / q d\left(r_{0}+2\right)} \\
\leq\left[C\left(r_{0}+2\right)^{2}\right]^{1 / 2\left(r_{0}+2\right)} \\
\quad \times\left(\int_{R^{N}}\left|f\left(v_{n}\right)\right|^{\left(2+r_{0}\right) q} d x\right)^{1 / q\left(r_{0}+2\right)} .
\end{aligned}
$$

Set $2+r_{1}=\left(2+r_{0}\right) d$. Then

$$
\begin{aligned}
\left(\int_{R^{N}}\left|f\left(v_{n}\right)\right|^{\left(2+r_{1}\right) q} d x\right)^{1 / q\left(r_{1}+2\right)} & \\
\leq & {\left[C\left(r_{0}+2\right)^{2}\right]^{1 / 2\left(r_{0}+2\right)} } \\
& \times\left(\int_{R^{N}}\left|f\left(v_{n}\right)\right|^{\left(2+r_{0}\right) q} d x\right)^{1 / q\left(r_{0}+2\right)} .
\end{aligned}
$$


Abstract and Applied Analysis

9

Inductively, we have

$$
\begin{aligned}
\left(\int_{R^{N}}\left|f\left(v_{n}\right)\right|^{\left(2+r_{k+1}\right) q} d x\right)^{1 / q\left(r_{k+1}+2\right)} & \\
\leq & {\left[C\left(r_{k}+2\right)^{2}\right]^{1 / 2\left(r_{k}+2\right)} } \\
& \times\left(\int_{R^{N}}\left|f\left(v_{n}\right)\right|^{\left(2+r_{k}\right) q} d x\right)^{1 / q\left(r_{k}+2\right)} \\
\leq & \prod_{i=0}^{k}\left[C\left(r_{i}+2\right)^{2}\right]^{1 / 2\left(r_{i}+2\right)} \\
& \times\left(\int_{R^{N}}\left|f\left(v_{n}\right)\right|^{\left(2+r_{0}\right) q} d x\right)^{1 / q\left(r_{0}+2\right)},
\end{aligned}
$$

where $\left(2+r_{i}\right)=d^{i}\left(2+r_{0}\right)(i=0,1, \ldots, k)$, and

$$
\begin{aligned}
& \prod_{i=0}^{k}\left[C\left(r_{i}+2\right)^{2}\right]^{\frac{1}{2\left(r_{i}+2\right)}} \\
& \quad=\exp \left\{\sum_{i=0}^{k} \frac{\ln \sqrt{C} d^{i}\left(r_{0}+2\right)}{d^{i}\left(r_{0}+2\right)}\right\} \\
& =\exp \left\{\sum_{i=0}^{k}\left[\frac{\ln \sqrt{C}\left(r_{0}+2\right)}{d^{i}\left(r_{0}+2\right)}+\frac{i \ln d}{d^{i}\left(r_{0}+2\right)}\right]\right\}
\end{aligned}
$$

is convergent as $k \rightarrow \infty$. Let $C_{k}=\prod_{i=0}^{k}\left[C\left(r_{i}+2\right)^{2}\right]^{1 / 2\left(r_{i}+2\right)}$. Then $C_{k} \rightarrow C_{\infty}>0$ as $k \rightarrow \infty$. Hence

$$
\left\|f\left(v_{n}\right)\right\|_{L^{\left(2+r_{0}\right) q d^{k+1}}} \leq C_{k}\left\|f\left(v_{n}\right)\right\|_{L^{2\left(2^{*}\right)}} .
$$

Let $k \rightarrow \infty$; by (65), we have

$$
\left\|f\left(v_{n}\right)\right\|_{L^{\infty}} \leq C_{\infty}\left\|f\left(v_{n}\right)\right\|_{L^{2\left(2^{*}\right)}} \leq C, \quad\|f(v)\|_{L^{\infty}} \leq C .
$$

Hence, by $\left(f_{9}\right)$ and (85), we have

$$
\begin{aligned}
\int_{R^{N}} V(x) & v_{n}^{2} d x \\
= & \int_{\left\{x:\left|v_{n}(x)\right| \leq 1\right\}} V(x) v_{n}^{2} d x \\
& +\int_{\left\{x:\left|v_{n}(x)\right|>1\right\}} V(x) v_{n}^{2} d x \\
\leq & \frac{1}{C} \int_{\left\{x:\left|v_{n}(x)\right| \leq 1\right\}} V(x) f^{2}\left(v_{n}\right) d x \\
& +\frac{1}{C} \int_{\left\{x:\left|v_{n}(x)\right|>1\right\}} V(x) f^{4}\left(v_{n}\right) d x \\
\leq & \frac{1}{C} \int_{\left\{x:\left|v_{n}(x)\right| \leq 1\right\}} V(x) f^{2}\left(v_{n}\right) d x \\
& +C \int_{\left\{x:\left|v_{n}(x)\right| \geq 1\right\}} V(x) f^{2}\left(v_{n}\right) d x \\
\leq & C \int_{R^{N}} V(x) f^{2}\left(v_{n}\right) d x .
\end{aligned}
$$

Since $\theta_{n} \rightarrow 0$, by (63)

$$
\theta_{n} \int_{R^{N}} V(x) v_{n} \psi \exp \left(-f\left(v_{n}\right)\right) d x \longrightarrow 0
$$

By $\left|\nabla\left(v_{n}-v\right)\right|^{2} \psi \exp \left(-f\left(v_{n}\right)\right) f^{\prime}\left(v_{n}\right) \geq 0$, one has

$$
\begin{gathered}
\limsup _{n \rightarrow \infty} \int_{R^{N}}-\left|\nabla v_{n}\right|^{2} \psi \exp \left(-f\left(v_{n}\right)\right) f^{\prime}\left(v_{n}\right) d x \\
\leq-\int_{R^{N}}|\nabla v|^{2} \psi \exp (-f(v)) f^{\prime}(v) d x .
\end{gathered}
$$

as $n \rightarrow \infty$. Moreover, notice that $v_{n} \rightarrow v$ in $E, v_{n} \rightarrow v$ in $L^{s}\left(R^{N}\right)$ for $s \in\left[2,2^{*}\right)$, and $v_{n}(x) \rightarrow v(x)$ a.e. $x \in R^{N}$; by Hölder inequality and Lebesgue theorem, we have

$$
\begin{gathered}
\int_{R^{N}} \exp \left(-f\left(v_{n}\right)\right) \nabla v_{n} \cdot \nabla \psi d x \\
\longrightarrow \int_{R^{N}} \exp (-f(v)) \nabla v \cdot \nabla \psi d x, \\
\int_{R^{N}} V(x) f\left(v_{n}\right) f^{\prime}\left(v_{n}\right) \psi \exp \left(-f\left(v_{n}\right)\right) d x \\
\longrightarrow \int_{R^{N}} V(x) f(v) f^{\prime}(v) \psi \exp (-f(v)) d x, \\
\int_{R^{N}} h\left(x, f\left(v_{n}\right)\right) f^{\prime}\left(v_{n}\right) \psi \exp \left(-f\left(v_{n}\right)\right) d x \\
\longrightarrow \int_{R^{N}} h(x, f(v)) f^{\prime}(v) \psi \exp (-f(v)) d x .
\end{gathered}
$$


Hence, from (87), we have

$$
\begin{aligned}
0 \leq & \int_{R^{N}} \exp (-f(v)) \nabla v \cdot \nabla \psi d x \\
& -\int_{R^{N}}|\nabla v|^{2} \psi \exp (-f(v)) f^{\prime}(v) d x \\
& +\theta_{n} \int_{R^{N}} V(x) v \psi \exp (-f(v)) d x \\
& +\int_{R^{N}} V(x) f(v) f^{\prime}(v) \psi \exp (-f(v)) d x \\
& -\int_{R^{N}} h(x, f(v)) f^{\prime}(v) \psi \exp (-f(v)) d x \\
= & \int_{R^{N}} \nabla v \cdot \nabla(\psi \exp (-f(v))) d x \\
& +\int_{R^{N}} V(x) f(v) f^{\prime}(v) \psi \exp (-f(v)) d x \\
& -\int_{R^{N}} h(x, f(v)) f^{\prime}(v) \psi \exp (-f(v)) d x .
\end{aligned}
$$

For any $\varphi \in E$ with $\varphi \geq 0$, by (85) we know that $\zeta:=$ $\varphi \exp (f(v)) \in E$. By Theorem 2.8 in [19], there exists a sequence $\left\{\psi_{n}\right\} \subset C_{0}^{\infty}\left(R^{N}\right)$ such that $\psi_{n} \geq 0$ and $\psi_{n} \rightarrow \zeta$ and $\psi_{n}(x) \rightarrow \zeta(x)$ for a.e. $x \in R^{N}$. Take $\psi=\psi_{n}$ in (91), and let $n \rightarrow \infty$; we have

$$
\begin{aligned}
0 \leq & \int_{R^{N}} \nabla v \cdot \nabla \varphi d x+\int_{R^{N}} V(x) f(v) f^{\prime}(v) \varphi d x \\
& -\int_{R^{N}} h(x, f(v)) f^{\prime}(v) \varphi d x .
\end{aligned}
$$

The opposite inequality can be obtained by taking $\phi=$ $\psi \exp \left(f\left(v_{n}\right)\right)$ and $\zeta=\varphi \exp (-f(v))$. Consequently,

$$
\begin{aligned}
\int_{R^{N}} \nabla v \cdot \nabla \varphi d x+\int_{R^{N}} V(x) f(v) f^{\prime}(v) \varphi d x \\
\quad-\int_{R^{N}} h(x, f(v)) f^{\prime}(v) \varphi d x=0, \quad \forall \varphi \in E .
\end{aligned}
$$

This shows that $v \in E$ is a critical point of $I$, and by taking $\varphi=f(v) / f^{\prime}(v) \in E$, one has

$$
\begin{gathered}
\int_{R^{N}}\left[1+\frac{2 f^{2}(v)}{1+2 f^{2}(v)}\right]|\nabla v|^{2} d x+\int_{R^{N}} V(x) f^{2}(v) d x \\
-\int_{R^{N}} h(x, f(v)) f(v) d x=0 .
\end{gathered}
$$

Finally, taking $\phi=f\left(v_{n}\right) / f^{\prime}\left(v_{n}\right)$ as the test function in (61), we have

$$
\begin{aligned}
\int_{R^{N}} & {\left[1+\frac{2 f^{2}\left(v_{n}\right)}{1+2 f^{2}\left(v_{n}\right)}\right]\left|\nabla v_{n}\right|^{2} d x+\theta_{n} \int_{R^{N}} V(x) \frac{v_{n} f\left(v_{n}\right)}{f^{\prime}\left(v_{n}\right)} d x } \\
& +\int_{R^{N}} V(x) f^{2}\left(v_{n}\right) d x \\
& -\int_{R^{N}} h\left(x, f\left(v_{n}\right)\right) f\left(v_{n}\right) d x=0 .
\end{aligned}
$$

Since

$$
\begin{gathered}
\int_{R^{N}} h\left(x, f\left(v_{n}\right)\right) f\left(v_{n}\right) d x \rightarrow \int_{R^{N}} h(x, f(v)) f(v) \mathrm{d} x, \\
\int_{R^{N}}\left[1+\frac{2 f^{2}\left(v_{n}\right)}{1+2 f^{2}\left(v_{n}\right)}\right]\left|\nabla\left(v_{n}-v\right)\right|^{2} d x \geq 0,
\end{gathered}
$$

by Fatou's Lemma, (63), (94), (95), up to subsequence, one has

$$
\begin{gathered}
\theta_{n} \int_{R^{N}} V(x) \frac{v_{n} f\left(v_{n}\right)}{f^{\prime}\left(v_{n}\right)} d x \rightarrow 0 \\
\int_{R^{N}}\left|\nabla v_{n}\right|^{2} d x \longrightarrow \int_{R^{N}}|\nabla v|^{2} d x \\
\int_{R^{N}} \frac{2 f^{2}\left(v_{n}\right)}{1+2 f^{2}\left(v_{n}\right)}\left|\nabla v_{n}\right|^{2} d x \longrightarrow \int_{R^{N}} \frac{2 f^{2}(v)}{1+2 f^{2}(v)}|\nabla v|^{2} d x \\
\int_{R^{N}} V(x) f^{2}\left(v_{n}\right) d x \longrightarrow \int_{R^{N}} V(x) f^{2}(v) d x .
\end{gathered}
$$

Hence $I_{\theta_{n}}\left(v_{n}\right) \rightarrow I(v)$ as $n \rightarrow \infty$. Set $w_{n}:=v_{n}-v \in E$. By $\left(f_{8}\right),\left(f_{12}\right)$, and $(85)$, one has

$$
\begin{aligned}
f^{2}\left(w_{n}\right)=f^{2}\left(2 \cdot \frac{w_{n}}{2}\right) & \leq C\left[\frac{1}{2} f^{2}\left(v_{n}\right)+\frac{1}{2} f^{2}(v)\right] \\
& \leq C\left[f^{2}\left(v_{n}\right)+f^{2}(v)\right] \leq C .
\end{aligned}
$$

Consequently, by $\left(f_{9}\right),(98)$, and Lemma 4 , one has

$$
\begin{aligned}
\int_{R^{N}} V(x)\left|w_{n}\right|^{2} d x & \\
= & \int_{\left\{x:\left|w_{n}\right| \leq 1\right\}} V(x)\left|w_{n}\right|^{2} d x \\
& +\int_{\left\{x:\left|w_{n}\right| \geq 1\right\}} V(x)\left|w_{n}\right|^{2} d x \\
\leq & C \int_{\left\{x:\left|w_{n}\right| \leq 1\right\}} V(x) f^{2}\left(w_{n}\right) d x \\
& +C \int_{\left\{x:\left|w_{n}\right| \geq 1\right\}} V(x) f^{4}\left(w_{n}\right) d x
\end{aligned}
$$




$$
\begin{aligned}
& \leq C \int_{\left\{x:\left|w_{n}\right| \leq 1\right\}} V(x) f^{2}\left(w_{n}\right) d x \\
& \quad+C \int_{\left\{x:\left|w_{n}\right| \geq 1\right\}} V(x) f^{2}\left(w_{n}\right) d x \\
& =C \int_{R^{N}} V(x) f^{2}\left(w_{n}\right) d x \longrightarrow 0
\end{aligned}
$$

as $n \rightarrow \infty$. Therefore, $v_{n} \rightarrow v$ in $E$. This completes the proof.

Theorem 6. Assume conditions $(V),\left(h_{1}\right)-\left(h_{3}\right)$ hold; then (1) has a weak solution.

Proof. First, we prove that, for each $\theta \in(0,1], I_{\theta}$ satisfy the Cerami condition. Indeed, let $\left\{v_{n}\right\} \subset E$ be an arbitrary Cerami sequence of $I_{\theta}$. Set $\phi=f\left(v_{n}\right) / f^{\prime}\left(v_{n}\right)$. Then $\|\phi\|_{E} \leq C\left\|v_{n}\right\|_{E}$. Similar to the proof of (63), we can prove that $\left\{v_{n}\right\}$ is bounded in $E$. Hence, by Lemma 3 , the sequence $\left\{v_{n}\right\}$ possesses a convergent subsequence in $E$. This shows that $I_{\theta}$ satisfy the Cerami condition.

Next, for any $\varepsilon>0$, by $\left(h_{1}\right),\left(h_{2}\right),\left(f_{3}\right)$, and $\left(f_{7}\right)$, there exists $C(\varepsilon)>0$ such that

$$
H(x, f(t)) \leq \varepsilon t^{2}+C(\varepsilon)|t|^{p / 2}
$$

for all $(x, t) \in R^{N} \times R$. For small $0<\rho \ll 1$, set

$$
S_{\rho}=\left\{v \in E:\|v\|_{E}=\rho\right\} .
$$

Then, from (101), for $v \in S_{\rho}$,

$$
\begin{aligned}
I_{\theta}(v)= & \frac{1}{2} \int_{R^{N}}\left[|\nabla v|^{2}+V(x) f^{2}(v)\right] d x \\
& +\frac{\theta}{2} \int_{R^{N}} V(x) v^{2} d x-\int_{R^{N}} H(x, f(v)) d x \\
\geq & \frac{\theta}{2} \int_{R^{N}}\left[|\nabla v|^{2}+V(x) v^{2}\right] d x \\
& -\varepsilon \int_{R^{N}} v^{2} d x-C(\varepsilon) \int_{R^{N}}|v|^{p / 2} d x \\
\geq & \frac{\theta}{2}\|v\|_{E}^{2}-\varepsilon a_{2}^{2}\|v\|_{E}^{2}-C(\varepsilon) a_{p / 2}^{p / 2}\|v\|_{E}^{p / 2} \\
\geq & \rho^{2}\left(\frac{\theta}{4}-C \rho^{(p-4) / 2}\right) \geq \delta>0
\end{aligned}
$$

for small $\varepsilon>0$ and $\rho>0$. Moreover, by $\left(h_{3}\right)$, for any $(x, z) \epsilon$ $R^{N} \times R$ with $|z| \geq r$, one has

$$
H(x, z) \geq c_{0}|z|^{\mu} .
$$

Since $\mu>4$, there exists a constant $4<\alpha<\min \left\{\mu, 2\left(2^{*}\right)\right\}$. Hence, by $\left(f_{5}\right)$, we have

$$
\lim _{|t| \rightarrow \infty} \frac{H(x, f(t))}{|t|^{\alpha / 2}}=\lim _{|t| \rightarrow \infty} \frac{H(x, f(t))}{|f(t)|^{\alpha}} \cdot \frac{|f(t)|^{\alpha}}{|t|^{\alpha / 2}}=+\infty
$$

uniformly in $x \in R^{N}$. Consequently, there exist constants $\tau>$ 1 such that

$$
H(x, f(t)) \geq|t|^{\alpha / 2}, \quad \forall|t| \geq \tau,
$$

for all $x \in R^{N}$. For any finite-dimensional subspace $\widetilde{E} \subset E$, by the equivalency of all norms in the finite-dimensional space, there is a constant $a>0$ such that

$$
\|v\|_{\alpha / 2} \geq a\|v\|_{E}, \quad \forall v \in \widetilde{E} .
$$

By $\left(h_{1}\right),\left(h_{2}\right)$, and (106), there exists a positive constant $C>0$ such that

$$
H(x, f(t)) \geq|t|^{\alpha / 2}-C t^{2}, \quad \forall(x, t) \in R^{N} \times R .
$$

Since $4<\alpha<2\left(2^{*}\right)$, by $\left(f_{3}\right),(107)$, and (108), we have

$$
\begin{aligned}
I_{\theta}(v)= & \frac{1}{2} \int_{R^{N}}\left[|\nabla v|^{2}+V(x) f^{2}(v)\right] d x \\
& +\frac{\theta}{2} \int_{R^{N}} V(x) v^{2} d x-\int_{R^{N}} H(x, f(v)) d x \\
\leq & \|v\|_{E}^{2}-\|v\|_{\alpha / 2}^{\alpha / 2}+C\|v\|_{2}^{2} \\
\leq & C\|v\|_{E}^{2}-a^{\alpha / 2}\|v\|_{E}^{\alpha / 2}
\end{aligned}
$$

for all $v \in \widetilde{E}$. Hence there exists a large $R>0$ such that $I_{\theta}<0$ on $\widetilde{E} \backslash B_{R}$. Set a fixed $e \in \widetilde{E}$ with $\|e\|_{E}=1$. For any fixed $T>\rho$, define the path $h_{T}:[0,1] \mapsto \widetilde{E} \subset E$ by $h_{T}(t)=t T e$. Then for large $T>0$, by (109), one has

$$
\begin{gathered}
I_{\theta}\left(h_{T}(1)\right) \leq C T^{2}-a^{\alpha / 2} T^{\alpha / 2}<0, \\
\left\|h_{T}(1)\right\|_{E}=T>\rho, \\
\sup _{t \in[0,1]} I_{\theta}\left(h_{T}(t)\right) \leq C T^{2}<+\infty .
\end{gathered}
$$

Hence by Theorem 2.2 with the Cerami condition in [20], $I_{\theta}$ possesses a critical value

$$
\begin{gathered}
c_{\theta}:=\inf _{\gamma \in \Gamma \in[0,1]} \max _{\theta} I_{\theta}(\gamma(t)) \geq \delta>0, \\
c_{\theta} \leq \sup _{t \in[0,1]} I_{\theta}\left(h_{T}(t)\right) \leq C T^{2},
\end{gathered}
$$

where

$$
\Gamma=\left\{\gamma \in C([0,1], E): \gamma(0)=0, \gamma(1)=h_{T}(1)\right\} .
$$

Consequently, by Theorem 5, we know that (1) has a weak solution. This completes the proof of Theorem 6 .

Remark 7. Let $v^{+}=\max \{v, 0\}$ and $v^{-}=\max \{-v, 0\}$. Set

$$
\begin{aligned}
I^{ \pm}(u)= & \frac{1}{2} \int_{R^{N}}\left[|\nabla v|^{2}+V(x) f^{2}(v)\right] d x \\
& -\int_{R^{N}} H\left(x, f\left(v^{ \pm}\right)\right) d x, \\
I_{\theta}^{ \pm}(v) & =\frac{1}{2} \theta \int_{R^{N}} V(x) v^{2} d x+I^{ \pm}(v)
\end{aligned}
$$

instead of $I(u)$ and $I_{\theta}(u)$, respectively. Then, under the conditions of Theorem 6 , we can obtain the existence of a positive solution and a negative solution for (1). 
Theorem 8. Assume conditions $(V),\left(h_{1}\right)-\left(h_{3}\right)$ hold. If $h(x, s)$ is odd in $s$, then (1) has a sequence $\left\{v_{m}\right\}$ of solutions such that $I\left(v_{m}\right) \rightarrow+\infty$.

Proof. Consider the eigenvalue of the operate $L=-\Delta+V$. By assumption $(V)$ and the compactness of the embedding $E \hookrightarrow L^{2}\left(R^{N}\right)$, we know that the spectrum $\sigma(L)=$ $\left\{\lambda_{1}, \lambda_{2}, \ldots, \lambda_{n} \cdots\right\}$ of $L$ with

$$
0<\lambda_{1}<\lambda_{2}<\cdots<\lambda_{n}<\cdots
$$

and $\lambda_{n} \rightarrow+\infty$ as $n \rightarrow+\infty$ (see page 3820 in [21]). Let $\varphi_{n}$ be the eigenfunction corresponding to $\lambda_{n}$. By regularity argument we know that $\varphi_{n} \in E$. Set $E_{n}=\operatorname{span}\left\{\varphi_{1}, \varphi_{2}, \ldots, \varphi_{n}\right\}$. Then we can decompose the space $E$ as $E=E_{n} \oplus W_{n}$ for $n=$ $1,2, \ldots$, where $W_{n}$ is orthogonal to $E_{n}$ in $E$. For $\rho>0$, set

$$
Q_{\rho}=\left\{v \in E: \int_{R^{N}}\left[|\nabla v|^{2}+V(x) f^{2}(v)\right] d x \leq \rho^{2}\right\} .
$$

By (109) there exists $r_{n}>0$ independent of $\theta$ such that

$$
I_{\theta}(v)<0, \quad \forall v \in \overline{E_{n} \backslash Q_{r_{n}}} .
$$

Set

$$
\begin{gathered}
D_{n}=E_{n} \cap Q_{r_{n}}, \\
G_{n}=\left\{\varphi \in C\left(D_{n}, E\right): \varphi \text { is odd and }\left.\varphi\right|_{\partial Q_{r_{n}} \cap E_{n}}=i d\right\}, \\
\Gamma_{j}=\left\{\varphi\left(\overline{D_{n} \backslash Q_{r_{n}}}\right): \varphi \in G_{n}, n \geq j,\right. \\
\left.A=-A \subset E_{n} \cap Q_{r_{n}} \text { is closed and } \gamma(A) \leq n-j\right\},
\end{gathered}
$$

where $\gamma(\cdot)$ is the genus. Let

$$
c_{j}(\theta)=\inf _{B \in \Gamma_{j}} \sup _{v \in B} I_{\theta}, \quad j=1,2 \ldots
$$

We have the following three facts (we refer the reader to [16] for their proofs).

Fact (1). For each $B \in \Gamma_{j}$, if $0<\rho<r_{n}$ for all $n \geq j$, then $B \cap \partial Q_{\rho} \cap W_{j-1} \neq \emptyset$.

Fact (2). There exist constants $\alpha_{j} \leq \beta_{j}$ such that $c_{j}(\theta) \in\left[\alpha_{j}, \beta_{j}\right]$ and $\alpha_{j} \rightarrow+\infty$ as $j \rightarrow+\infty$.

Fact (3). $c_{j}(\theta), j=1,2, \ldots$ are critical values of $I_{\theta}$.

Consequently, Theorem 8 follows from Theorem 5 and the above Facts (2)-(3). This completes the proof.

Corollary 9. If the following conditions $\left(h_{4}\right)$ and $\left(h_{5}\right)$ are used in place of $\left(h_{3}\right)$; then the conclusions of Theorem 5, Theorem 6 , and Theorem 8 hold:

$\left(h_{4}\right) \lim _{|s| \rightarrow+\infty} \inf H(x, s)>0$ uniformly in $x \in \mathbb{R}^{N}$,

$\left(h_{5}\right)$ there exist $\mu>4$ and $\tau>0$ such that

$$
\mu H(x, s) \leq h(x, s) s
$$

for all $x \in \mathbb{R}^{N}$ and $|s| \geq \tau$.
Proof. By $\left(h_{4}\right)$, there are constants $\lambda>0$ and $r_{1}>0$ such that whenever $|s| \geq r_{1}$, one has

$$
H(x, s)>\lambda, \quad \forall x \in \mathbb{R}^{N} .
$$

Set $r=\max \left\{\tau, r_{1}\right\}$. Then, by $\left(h_{5}\right)$,

$$
\begin{gathered}
c_{0}:=\inf _{x \in \mathbb{R}^{N},|s|=r} H(x, s) \geq \lambda>0, \\
\mu H(x, s) \leq h(x, s) s
\end{gathered}
$$

for all $x \in \mathbb{R}^{N}$ and $|s| \geq r$. Therefore, condition $\left(h_{3}\right)$ holds. This completes the proof.

\section{Acknowledgment}

This work was supported partially by the National Natural Science Foundation of China (11261070).

\section{References}

[1] S. Kurihura, "Large-amplitude quasi-solitons in superfluid films," Journal of the Physical Society of Japan, vol. 50, pp. 32623267, 1981.

[2] E. W. Laedke, K. H. Spatschek, and L. Stenflo, "Evolution theorem for a class of perturbed envelope soliton solutions," Journal of Mathematical Physics, vol. 24, no. 12, pp. 2764-2769, 1983.

[3] A. G. Litvak and A. M. Sergeev, "One dimensional collapse of plasma waves," JETP Letters, vol. 27, pp. 517-520, 1978.

[4] A. Nakamura, "Damping andmod ification of exciton solitary waves," Journal of the Physical Society of Japan, vol. 42, pp. 18241835, 1977.

[5] M. Porkolab and M. V. Goldman, "Upper-hybrid solitons and oscillating-two-stream instabilities," The Physics of Fluids, vol. 19, no. 6, pp. 872-881, 1976.

[6] J. Liu and Z. Q. Wang, "Soliton solutions for quasilinear Schrödinger equations. I," Proceedings of the American Mathematical Society, vol. 131, no. 2, pp. 441-448, 2003.

[7] M. Poppenberg, K. Schmitt, and Z. Q. Wang, "On the existence of soliton solutions to quasilinear Schrödinger equations," Calculus of Variations and Partial Differential Equations, vol. 14, no. 3, pp. 329-344, 2002.

[8] J. M. B. do Ó, O. H. Miyagaki, and S. H. M. Soares, "Soliton solutions for quasilinear Schrödinger equations with critical growth," Journal of Differential Equations, vol. 248, no. 4, pp. 722-744, 2010

[9] M. Colin and L. Jeanjean, "Solutions for a quasilinear Schrödinger equation: a dual approach," Nonlinear Analysis: Theory, Methods \& Applications, vol. 56, no. 2, pp. 213-226, 2004.

[10] J. Q. Liu, Y. Q. Wang, and Z. Q. Wang, "Soliton solutions for quasilinear Schrödinger equations. II," Journal of Differential Equations, vol. 187, no. 2, pp. 473-493, 2003.

[11] J. M. B. do Ó, O. H. Miyagaki, and S. H. M. Soares, "Soliton solutions for quasilinear Schrödinger equations: the critical exponential case," Nonlinear Analysis: Theory, Methods \& Applications, vol. 67, no. 12, pp. 3357-3372, 2007.

[12] J. Q. Liu, Y. Q. Wang, and Z. Q. Wang, "Solutions for quasilinear Schrödinger equations via the Nehari method," Communications in Partial Differential Equations, vol. 29, no. 5-6, pp. 879901, 2004. 
[13] D. Ruiz and G. Siciliano, "Existence of ground states for a modified nonlinear Schrödinger equation," Nonlinearity, vol. 23, no. 5, pp. 1221-1233, 2010.

[14] J. Q. Liu, Z. Q. Wang, and Y. X. Guo, "Multibump solutions for quasilinear elliptic equations," Journal of Functional Analysis, vol. 262, no. 9, pp. 4040-4102, 2012.

[15] X. Q. Liu, J. Liu, and Z. Q. Wang, "Ground states for quasilinear Schrödinger equations with critical growth," Calculus of Variations and Partial Differential Equations, vol. 46, no. 3-4, pp. 641669, 2013.

[16] X. Q. Liu, J. Q. Liu, and Z. Q. Wang, "Quasilinear elliptic equations via perturbation method," Proceedings of the American Mathematical Society, vol. 141, no. 1, pp. 253-263, 2013.

[17] W. M. Zou and M. Schechter, Critical Point Theory and Its Applications, Springer, New York, NY, USA, 2006.

[18] M. Willem, Minimax Theorems, Progress in Nonlinear Differential Equations and their Applications, 24, Birkhäuser, Boston, Mass, USA, 1996.

[19] S. Wang, Introductions of Sobolev Spaces and Partial Differential Equation, Scientific Publishing House in China, 2009.

[20] P. H. Rabinowitz, Minimax Methods in Critical Point Theory with Applications to Differential Equations, vol. 65 of CBMS Regional Conference Series in Mathematics, American Mathematical Society, Providence, RI, USA, 1986.

[21] A. Szulkin and T. Weth, "Ground state solutions for some indefinite variational problems," Journal of Functional Analysis, vol. 257, no. 12, pp. 3802-3822, 2009. 


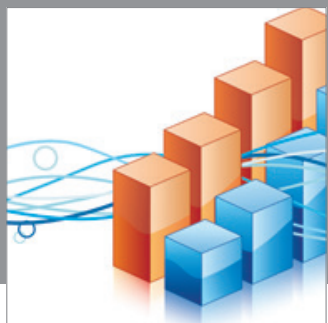

Advances in

Operations Research

mansans

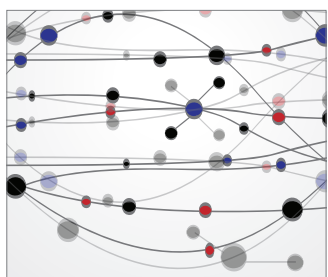

The Scientific World Journal
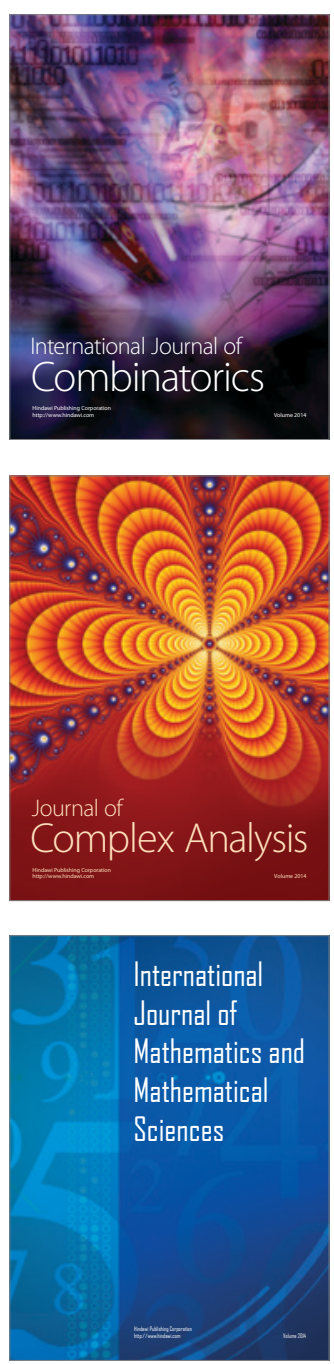
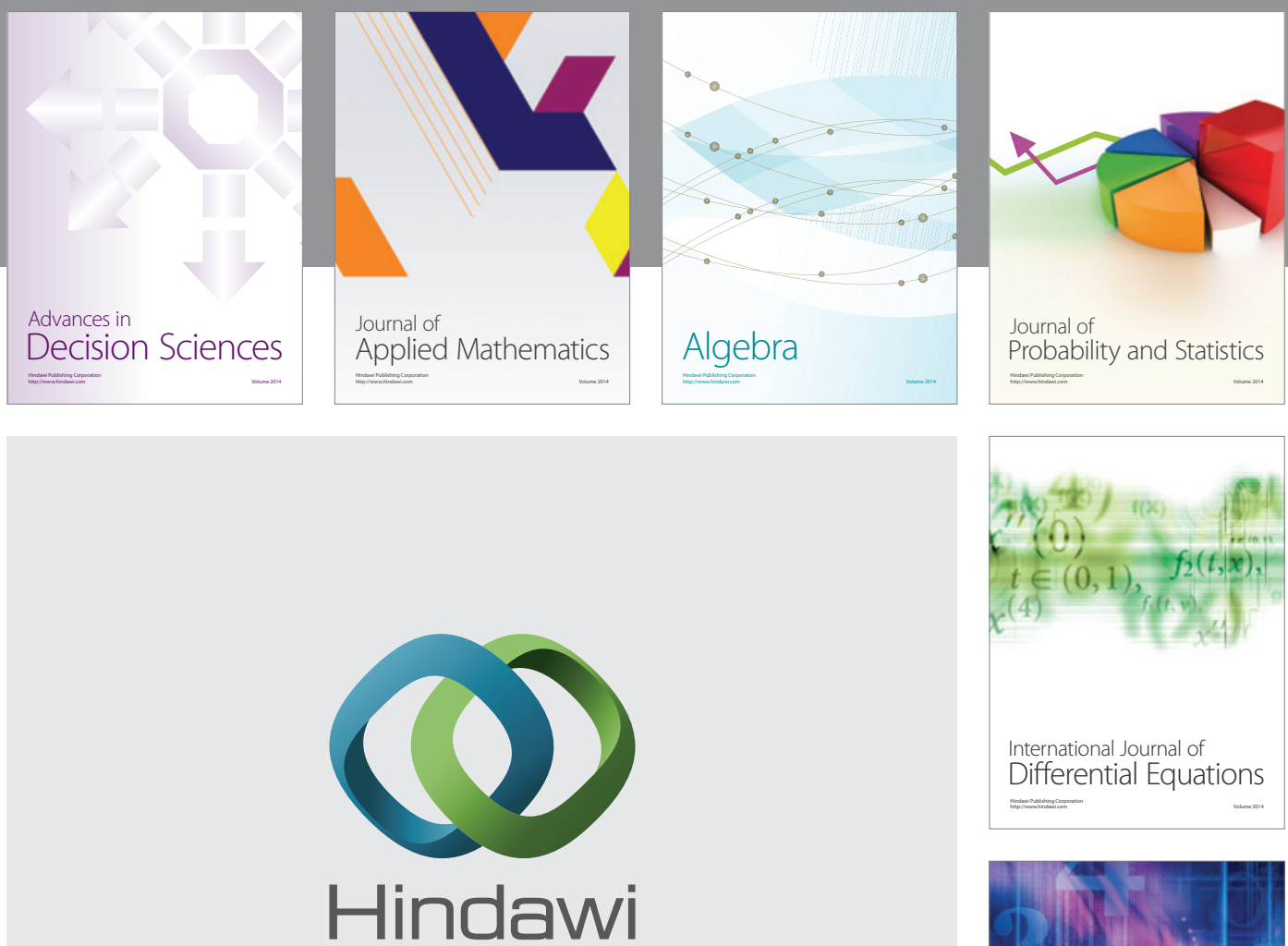

Submit your manuscripts at http://www.hindawi.com
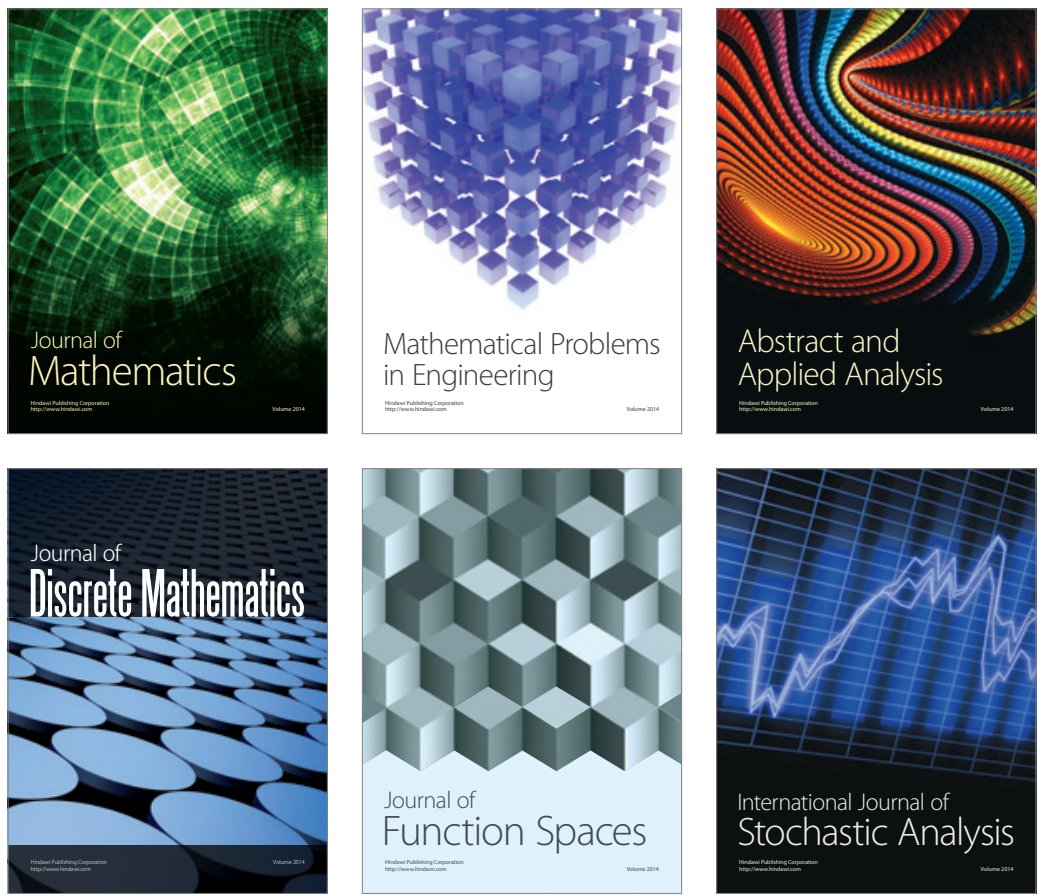

Journal of

Function Spaces

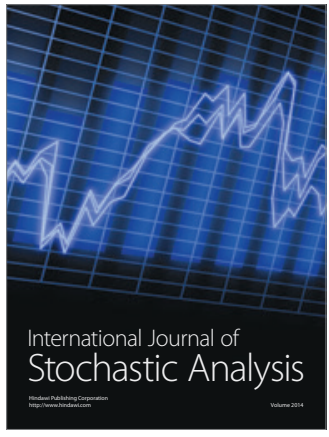

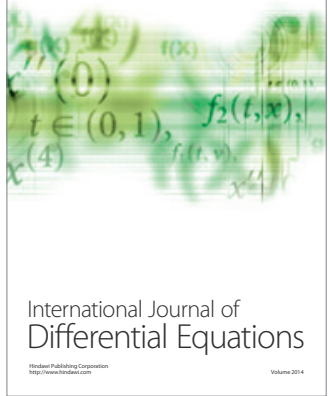
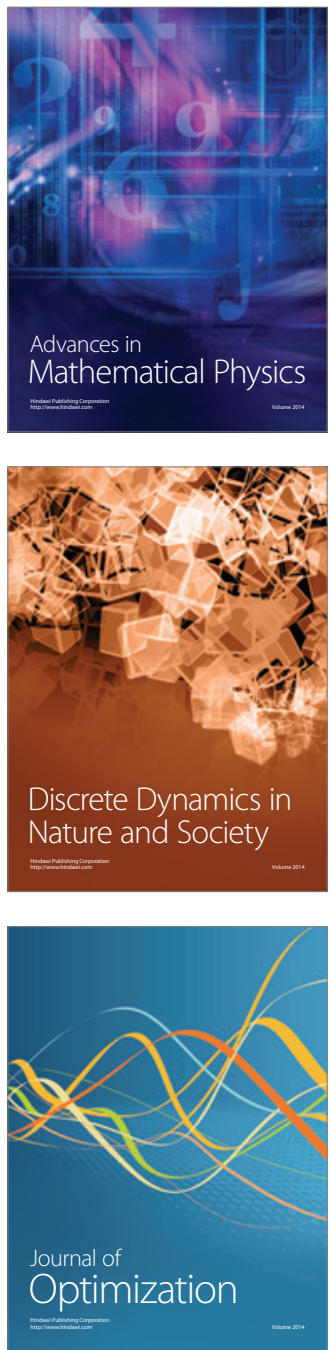